\title{
Glitazone - eine neue Substanzklasse in der Behandlung des Typ-2-Diabetes
}

Die Glitazone (Thiazolidindione) stellen eine neue Substanzklasse für die Therapie des Typ-2-Diabetes dar. Im Gegensatz zu den herkömmlichen oralen Antidiabetika besitzen die Glitazone eine direkte Wirkung auf die Insulinsignalübertragung an den insulinsensitiven Zielzellen. Über die Modulation des nuklären Rezeptors PPAR me-proliferator activated Receptor) erfolgt eine Aktivierung der frühen Insulin-Signalkaskade und eine Steigerung des Glukosetransports. Neben der Verbesserung der Insulinresistenz, des Glukose-Stoffwechsels sowie Inhibierung der hepatischen Glukoneogenese konnte auch eine Senkung der freien Fettsäuren, der Triglyzeride sowie ein Anstieg des HDL-Cholesterins gezeigt werden. Zusätzlich wurden günstige Effekte auf Blutdruck, Gefäße und Gerinnung nachgewiesen. $\mathrm{Zu}$ den Nebenwirkungen gehören vor allem eine leichte Gewichtszunahme, Anämie sowie periphere Ödeme, eine Behandlung herzinsuffizienter Patienten (einschließlich NYHA I) ist kontraindiziert.

\section{Wirkungsmechanismus}

Bei Glitazonen handelt es sich um Agonisten des nukleären Rezeptors PPAR $\gamma$. PPAR $\gamma$ liegt als Heterodimer mit dem Retinsäure-Rezeptor vor und bindet an bestimmte "PPAR responsible Elemente" in der Promotorregion von Zielgene und dann kann eine Gentranskription stattfinden. Die Aktivierung von PPAR $\gamma$ fördert die Differenzierung verschiedener Zellen einschließlich der Adipozytendifferenzierung und die Induktion gluco-regulatorischer Proteine. In verschiedenen Studien wurde unter Glitazonen signifikant niedrigere Nüchternund postprandiale Glukose- und Insulinspiegel und eine Verbesserung des Insulin-stimulierten Glukosetransports gemessen. Glitazone wurden ebenfalls zur Behandlung der Insulinresistenz bei Patienten mit Adipositas, gestörter Glukosetoleranz (IGT) oder polyzystischem Ovarialsyndrom eingesetzt, und

Dtsch. Med. Wschr. 2001; 126: 951-953 (c) Georg Thieme Verlag Stuttgart · New York eine Verbesserung der Insulinsensitivität konnte ebenfalls gezeigt werden.

Troglitazon, Rosiglitazon und Pioglitazon. besitzen eine gemeinsame Thiazolidin-2-4-Dion Struktur, unterscheiden sich jedoch in ihren Seitenketten. Dadurch erklären sich ihre Unterschiede in der Bioverfügbarkeit, ihrer antihyperglykämischen Effekte und Nebenwirkungen. Diese Substanzen wurden ursprünglich zur Inhibierung zur Lipidperoxidation aus einem Vitamin E Motiv und einem Fibratanteil entwickelt (Abb.1).

\section{Troglitazon}

Inzwischen sind über eine Million Patienten mit Glitazonen therapiert worden. In Deutschland wurde die Zulassung von Troglitazon gestoppt, nachdem in den USA einzelne aber sehr schwerwiegende Leberschäden aufgetreten sind. Troglitazon besitzt einen $\alpha$ Tocopherol-Ring, der möglicherweise für die Lebertoxizität verantwortlich ist (Abb.1). Etwa 2\% aller behandelten Patienten entwickelten einen Anstieg der Transaminasen und mindestens $25 \mathrm{~Pa}-$ tienten mit schwerer Leberschädigung sind bisher bekannt (3).

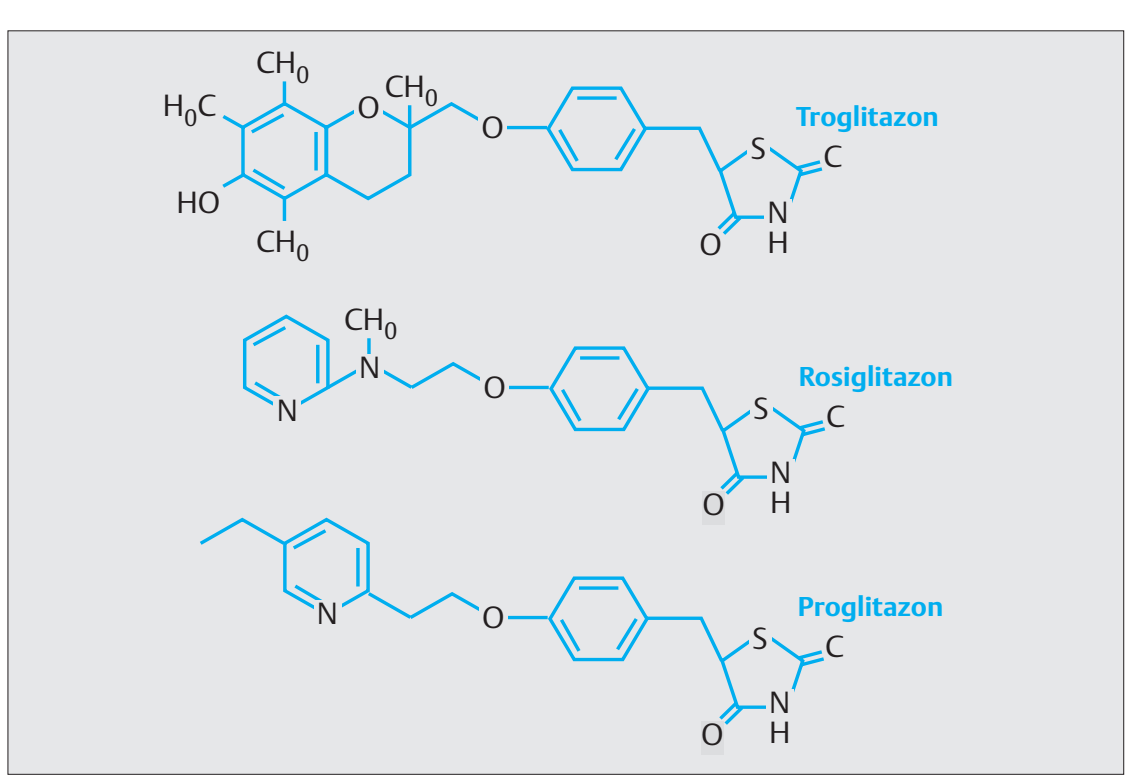

Abb. 1 Chemische Struktur der Glitazon-Derivate.

\section{Rosiglitazon}

Rosiglitazon ist etwa 100 -fach potenter als Troglitazon und wird deshalb in geringeren Dosierungen verabreicht. Rosiglitazon besitzt eine sehr hohe Bioverfügbarkeit (bei ungefähr 99\%) und wird im Gegensatz zu Troglitazon nicht über das 3A4-Isoenzym des hepatischen $\mathrm{Cy}-$ tochrom P450-Systems verstoffwechselt, sondern über CYP2C8 und mit geringer Beteiligung über CYP2C9 metabolisiert (2). Die Ausscheidung erfolgt zu zwei Drittel renal, über den Faeces werden nur etwa $25 \%$ eliminiert. Eine Anpassung der Dosis bei eingeschränkter Nierenfunktion ist nicht erforderlich, und auch das Alter der Patienten hat keine signifikanten Einfluss auf die Pharmakokinetik. Bislang sind in kontrollierten Studien mehr als 5000 Patienten mit Rosiglitazon behandelt worden. Kürzlich wurden zwei Fallberichte veröffentlicht, die unter Rosiglitazon einen Leberzellschaden entwickelten, der sich nach Absetzen wieder zurückbildete $(1,4)$. In einer klinischen Studie über 26 Wochen wurden 493 Patienten mit Typ-2-Diabetes einmal täglich mit Rosiglitazon behandelt. Die Nüchternblutzuckerspiegel konnten um $57,7 \mathrm{mg} / \mathrm{dl}$ unter $2 \mathrm{mg}$ und um $75,9 \mathrm{mg} / \mathrm{dl}$ unter $4 \mathrm{mg}$ Rosiglitazon

Redaktion: K. Mohr, Bonn

S. Herzig, Köln

AS 35 
gesenkt werden. Der $\mathrm{HbA}_{1 \mathrm{c}}$ wurde um 1,2 bzw. 1,5 Prozentpunkte gegenüber Placebo gesenkt. Ebenfalls kam es zu einem signifikanten Anstieg des HDLs und einer Reduktion der freien Fettsäuren sowie der Triglyceridspiegel (5). Im Rahmen einer 52-Wochen-Studie konnte unter Rosiglitazon eine 26,4\% Abnahme der Albuminausscheidung im Vergleich zu 9,1\% unter der Behandlung mit Glibenclamid gezeigt werden (7). In der gleichen Studie konnte der diastolische Blutdruck signifikant um 2,3 mmHg gesenkt werden, während er unter Glibenclamid um 0,7 mmHg anstieg. Als $\mathrm{Ne}-$ benwirkungen treten periphere Ödeme und Anämien sowie eine leichte $\mathrm{Ge}$ wichtszunahme von $1-3 \mathrm{~kg}$ auf. Durch die Flüssigkeitsretention kann eine Herzinsuffizienz ausgelöst oder eine bestehende verschlimmert werden. Für die Behandlung mit Glitazonen besteht deshalb eine Kontraindikation für Patienten mit Herzinsuffizienz einschließlich NYHA Stadium I.

\section{Pioglitazon}

Pioglitazon wird nach oraler Einnahme rasch und zu einem hohen Prozentsatz (85-96\%) resorbiert. Die Elimination erfolgt zu 55\% über die Faeces und zu $45 \%$ über den Urin. Pioglitazon wird einmal täglich verabreicht. Die Einmalgabe von $30 \mathrm{mg}$ bzw. $45 \mathrm{mg}$ Pioglitazon gegen Placebo über 12 Wochen resultierte in einem $\mathrm{HbA}_{1 \mathrm{c}}$-Abfall von 1,0 bzw. 1,4 Prozentpunkten und einer Reduktion der Nüchternblutzucker von $28-33 \mathrm{mg} / \mathrm{dl}$ bzw. $41-46 \mathrm{mg} / \mathrm{dl}$ (8). Es kam ebenfalls zu einem signifikanten Anstieg des HDLs, einer Reduktion der freien Fettsäuren und der Triglyceridspiegel. Auch bei eingeschränkter Nierenfunktion und bei älteren Patienten kann Pioglitazon verabreicht werden. Genau wie für Rosiglitazon lassen sich unter der Therapie mit Pioglitazon hepatotoxische Effekte nicht mit Sicherheit ausschließen. Auch eine leichte Gewichtszunahme, Anämie und periphere Ödeme wurden als Nebenwirkungen beobachtet, und es besteht eine Kontraindikation für Patienten mit Herzinsuffizienz einschließlich NYHA Stadium I. Bei allen Substanzen besteht kein Hypoglykämie-Risiko und keine Gefahr der Laktatazidose. Die verschiedenen Eigenschaften der Substanzen sind in Tab. 1 nochmals zusammengefasst.

Tab.1 Eigenschaften von Troglitazon, Rosiglitazon und Pioglitazon.

\begin{tabular}{llll}
\hline & Troglitazon & Rosiglitazon & Pioglitazon \\
\hline Dosis $(\mathrm{mg} / \mathrm{dl})$ & $200-600$ & $4-8$ & $15-45$ \\
Senkung des Nüchtern-BZ $(\mathrm{mg} / \mathrm{dl})$ & $25-40$ & $30-50$ & $30-50$ \\
Senkung des Insulinspiegels $(\mathrm{uU} / \mathrm{ml})$ & $2-5$ & 3 & nicht bekannt \\
Verminderung des HbA ${ }_{1 \mathrm{c}}(\%)$ & 0,7 & 1,0 & 1,0 \\
Effekte auf Serum-Lipidspiegel & TG $\downarrow, \mathrm{HDL} \uparrow, \mathrm{LDL}(\uparrow)$ & $\mathrm{FFA} \downarrow, \mathrm{TG} \downarrow, \mathrm{HDL} \uparrow$ & $\mathrm{TG} \downarrow, \mathrm{HDL} \uparrow$ \\
Hepatotoxizität & ja & nein * & nein \\
CK-Erhöhung & nicht bekannt & nein & ja \\
Herzhypertrophie & nein & nein & nein \\
Interaktion mit CPY 3A4 & ja & nein & ja
\end{tabular}

BZ: Blutzucker, TG: Triglyceride, FFA: freie Fettsäuren, CPY 3A4: Cytochrom P450 Isoform 3A4, * 2 Fallberichte über Leberschädigung

\section{Kombination mit anderen oralen Antidiabetika}

348 Patienten mit Typ-2-Diabetes, die unter maximaler Dosis von $2,5 \mathrm{~g}$ Metformin täglich nicht gut eingestellt waren, wurden in eine 26-wöchige Doppelblindstudie aufgenommen. Die zusätzliche Gabe von einmal täglich $4 \mathrm{mg}$ oder $8 \mathrm{mg}$ Rosiglitazon zu Metformin verbesserte die Nüchternblutzucker um weitere 39,8 und $52,9 \mathrm{mg} / \mathrm{dl}$ und den $\mathrm{HbA}_{1 \mathrm{c}}$ um 1,0 bzw. 1,2 Prozentpunkte (3). 574 Patienten, die unter der Therapie mit Sulfonylharnstoffen schlecht eingestellt waren, erhielten zusätzlich über 26 Wochen Rosiglitazon. Die Gabe von täglich 2 oder $4 \mathrm{mg}$ Rosiglitazon zusätzlich zu Glibenclamid führte im Vergleich zu Placebo zu einer weiteren Senkung des $\mathrm{HbA}_{1 \mathrm{c}}$ (0,6 und 1,0 Prozentpunkte). Parallel wurde ein Verbesserung der Nüchternblutzucker um 24,3 und $44 \mathrm{mg} / \mathrm{dl}$ im Vergleich zur alleinigen Therapie mit Sulfonylharnstoff gemessen (7).

\section{Indikationen}

Die Behandlung mit Glitazonen ist zur Zeit nur angezeigt zur oralen Kombinationsbehandlung von Patienten mit Typ-2-Diabetes, deren Blutzuckerkontrolle, trotz einer oralen Monotherapie von Metformin oder Sulfonylharnstoffen, unzureichend ist. Eine Kombination mit Insulin ist bisher nicht zugelassen. Es wurden Effekte auf die Zellproliferation und Inflammation unter Glitazonen beschrieben. Unterschiedliche Aussagen wurden über den Einfluss von Glitazonen auf Kolontumore veröffentlicht. Vorerst sollte aufgrund der kontroversen Daten der Einsatz von
Glitazonen nicht bei Patienten mit Tumorerkrankungen des Kolons erfolgen. Grundsätzlich sollten bei dieser neuen Substanzklasse die Transaminasen vor Beginn der Therapie und während der Therapie gemessen werden. Bei Werten dreifach oberhalb der Norm sollte das Medikament abgesetzt werden. Bei Unsicherheit über das Vorliegen einer Herzinsuffizienz (NYHA I) ist eine Echokardiographie indiziert.

Glitazone stellen eine neue ergänzende orale Therapieform für die Behandlung der Insulinresistenz und des Typ-2-Diabetes dar, die pathophysiologisch sinnvoll erscheint.

\section{Literatur}

1 Al-Salman J, Arjomand H, Kemp DG, Mittal M. Hepatocellular injury in a patient receiving rostiglitazone. A case report. Ann Intern Med 2000; 132: 121-124

2 Baldwin SJ, Clarke SE, Chenery RJ. Characterization of the cytochrome P450 enzymes involved in the in vitro metabolism of rosiglitazone.. Br J Clin Pharmacol 1999; 48: 424 432

3 Fonseca V, Rosenstock J, Patwardhan R, Salzman A. Effect of metformin and rosiglitazone combination therapy in patients with type 2 diabetes mellitus: A randomized controlled trial.. Jama 2000; 283: 1695-1702

4 Freid J, Everitt D, Boscia J. Rosiglitazone and hepatic failure. Ann Intern Med 2000; 132: 164

5 Raskin P, Rappaport EB, Cole ST, Yan Y, Patwardhan R, Freed MI. Rosiglitazone shortterm monotherapy lowers fasting and postprandial glucose in patients with type II diabetes. Diabetologia 2000; 43: 278-284

6 Watkins PB, Whitcomb RW. Hepatic dysfunction associated with troglitazone.. N Engl J Med 1998; 338: 916-917

7 Wolffenbuttel BH, Gomis R, Squatrito S, Jones NP, Patwardhan RN. Addition of lowdose rosiglitazone to sulphonylurea therapy improves glycaemic control in Type 2 diabetic patients.. Diabet Med 2000; 17: 40-47

8 Yamasaki Y, Kawamori R, Wasada T et al. Pioglitazone (AD-4833) ameliorates insulin 
resistance in patients with NIDDM. AD-4833 Glucose Clamp Study Group, Japan.. Tohoku J Exp Med 1997; 183: 173-183

\section{Dr. Petra Algenstaedt}

Medizinische Kernklinik und Poliklinik

Universitätsklinikum

Hamburg-Eppendorf

Martinistraße 52

20246 Hamburg

Dr. Andreas Hamann

Abteilung für Innere Medizin I der

Medizinischen Kernklinik und

Poliklinik,

Universität Heidelberg

Bergheimerstr. 58

69115 Heidelberg 